Article

\title{
Coupling between Spin and Charge Order Driven by Magnetic Field in Triangular Ising System $\mathrm{LuFe}_{2} \mathrm{O}_{4+\delta}$
}

\author{
Lei Ding ${ }^{1, *(1)}$, Fabio Orlandi ${ }^{1}$ (1) , Dmitry D. Khalyavin ${ }^{1}$, Andrew T. Boothroyd ${ }^{2}$, \\ Dharmalingam Prabhakaran ${ }^{2}$, Geetha Balakrishnan ${ }^{3}$ and Pascal Manuel ${ }^{1, *}$ \\ 1 ISIS Pulsed Neutron Facility, Rutherford Appleton Laboratory, Harwell Oxford, Didcot OX11 0QX, UK; \\ fabio.orlandi@stfc.ac.uk (F.O.); dmitry.khalyavin@stfc.ac.uk (D.D.K.) \\ 2 Department of Physics, Oxford University, Clarendon Laboratory, Parks Road, Oxford OX1 3PU, UK; \\ Andrew.Boothroyd@physics.ox.ac.uk (A.T.B.); dharmalingam.prabhakaran@physics.ox.ac.uk (D.P.) \\ 3 Department of Physics, University of Warwick, Coventry CV4 7AL, UK; G.Balakrishnan@warwick.ac.uk \\ * Correspondence: lei.ding@stfc.ac.uk (L.D.); pascal.manuel@stfc.ac.uk (P.M.); Tel.: +44-123-544-6290 (P.M.)
}

Received: 19 January 2018; Accepted: 31 January 2018; Published: 6 February 2018

\begin{abstract}
We present a study of the magnetic-field effect on spin correlations in the charge ordered triangular Ising system $\mathrm{LuFe}_{2} \mathrm{O}_{4+\delta}$ through single crystal neutron diffraction. In the absence of a magnetic field, the strong diffuse neutron scattering observed below the Neel temperature $\left(\mathrm{T}_{\mathrm{N}}=240 \mathrm{~K}\right)$ indicates that $\mathrm{LuFe}_{2} \mathrm{O}_{4+\delta}$ shows short-range, two-dimensional (2D) correlations in the $\mathrm{FeO}_{5}$ triangular layers, characterized by the development of a magnetic scattering rod along the $1 / 3$ $1 / 3 \mathrm{~L}$ direction, persisting down to $5 \mathrm{~K}$. We also found that on top of the 2D correlations, a long range ferromagnetic component associated with the propagation vector $\mathbf{k}_{\mathbf{1}}=0$ sets in at around $240 \mathrm{~K}$. On the other hand, an external magnetic field applied along the c-axis effectively favours a three-dimensional (3D) spin correlation between the $\mathrm{FeO}_{5}$ bilayers evidenced by the increase of the intensity of satellite reflections with propagation vector $\mathbf{k}_{2}=(1 / 3,1 / 3,3 / 2)$. This magnetic modulation is identical to the charge ordered superstructure, highlighting the field-promoted coupling between the spin and charge degrees of freedom. Formation of the 3D spin correlations suppresses both the rod-type diffuse scattering and the $\mathbf{k}_{\mathbf{1}}$ component. Simple symmetry-based arguments provide a natural explanation of the observed phenomenon and put forward a possible charge redistribution in the applied magnetic field.
\end{abstract}

Keywords: single crystal neutron diffraction; magnetic structure; diffuse scattering; charge order; spin frustration

\section{Introduction}

The layered oxide $\mathrm{LuFe}_{2} \mathrm{O}_{4}$, bearing significant spin and charge frustration in a triangular lattice, has attracted wide interest thanks to reported high-temperature multiferroic properties, pivotal for technological applications in spintronics [1-6]. Such properties have been related to the charge ordering of $\mathrm{Fe}^{2+}$ and $\mathrm{Fe}^{3+}$ cations, which appears as a three-dimensional (3D) long range ordering below $320 \mathrm{~K}$ [2]. An apparent coupling between polarization and magnetism has also been previously proposed based on the observation of significant changes in the electric polarization when spin ordering sets in at $\mathrm{T}_{\mathrm{N}}=240 \mathrm{~K}$ [3]. Although the polar nature of the charge ordering was not confirmed by the later structural study [7], $\mathrm{LuFe}_{2} \mathrm{O}_{4}$ remains a focus of intensive investigations as a model frustrated system.

The parent structure of rhombohedral $\mathrm{LuFe}_{2} \mathrm{O}_{4}$ (space group $R-3 m$ ) (Figure 1) is characterized by the stacking of three $\mathrm{FeO}_{5}$ face-sharing bipyramidal layers along the c-axis separated by $\mathrm{LuO}_{2}$ rock salt layers $[1,2]$. The resulting iron sublattice arrangement consists of three stacked triangular bilayers with an equal amount of $\mathrm{Fe}^{2+}$ and $\mathrm{Fe}^{3+}$ in the unit cell, and this makes the system geometrically frustrated 
in both the charge and spin channels. Even though intensive experimental and theoretical efforts to clarify the underlying pattern of spin and charge order have been made, its exact nature is still controversial [8].

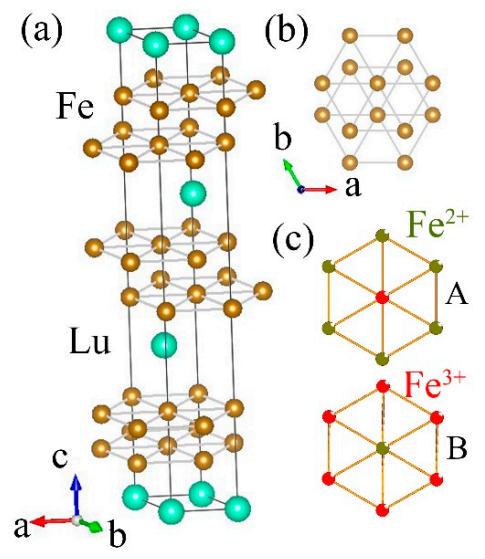

Figure 1. (a) The crystal structure of $\mathrm{LuFe}_{2} \mathrm{O}_{4}$. (b) Two adjacent triangular $\mathrm{FeO}_{5}$ layers shifted by $\sqrt{3} / 3$ a within the $(a b)$ plane. (c) The $\mathrm{Fe}^{2+}-(\mathrm{A})$ and $\mathrm{Fe}^{3+}$-rich (B) monolayer charge models. Oxygen ions are omitted for clarity.

A physical picture of the charge order, overcoming the charge frustration in the triangular layers has been proposed in the so called AB model [2,3,5] where A represents an upper iron layer with $\mathrm{Fe}^{3+}$ cations surrounded by a honeycomb lattice of $\mathrm{Fe}^{2+}$ cations and a lower $\mathrm{B}$ layer has the opposite $\mathrm{Fe}^{2+} / \mathrm{Fe}^{3+}$ arrangement (see Figure $1 \mathrm{c}$ ). Whereas the $\mathrm{AB}$ model has been used to account for the ferroelectricity observed in $\mathrm{LuFe}_{2} \mathrm{O}_{4}$ due to the net electric dipole moment along the $c$-axis [2,3], X-ray scattering experiments have suggested an antiferroelectric AB-BA bilayer stacking [6,9]. Later, several investigations have found that ferroelectric order can be induced based on this antiferroelectric model when an external electric field is applied [10-12]. A charge order model with AA-BB stacking has also been recently demonstrated through X-ray diffraction study, inconsistent with charge-ordering-based ferroelectricity [7].

On the other hand, the exact spin configuration is also under debate. The magnetic properties of $\mathrm{LuFe}_{2} \mathrm{O}_{4}$ are governed by the Ising character of the iron spin in the triangular lattice essentially due to spin-orbit coupling [8,13]. Both two-dimensional (2D) [1] and 3D magnetic structures [12,14] have been previously found in different samples, reflecting the important role played by oxygen stoichiometry [15-18]. In addition, two magnetic phase transitions have been observed by Christianson et al. via single crystal neutron diffraction study. The first transition, observed below $\mathrm{T}_{\mathrm{N}}=240 \mathrm{~K}$, is characterized by finite magnetic correlations within ferrimagnetic (FIM) monolayers stacked ferromagnetically along the $c$ direction; while at the second transition $\left(\mathrm{T}_{\mathrm{L}}=175 \mathrm{~K}\right)$ a significant broadening of specific magnetic reflections is observed, indicative of an additional decrease in the magnetic correlation length [14,19]. Moreover, a metamagnetic state in the vicinity of $\mathrm{T}_{\mathrm{N}}$, consisting of nearly degenerate FIM and antiferromagnetic (AFM) spin order has been proposed [20]. A distinct FIM spin configuration on the bilayers based on the $\mathrm{AB}$ charge order model, where all $\mathrm{Fe}^{2+}$ spins in the adjacent monolayers are $\mathrm{FM}$, while $\mathrm{Fe}^{3+}$ spins are antiparallel to $\mathrm{Fe}^{2+}$ rich layers and nearest neighbour $\mathrm{Fe}^{3+}$ spins in the $\mathrm{Fe}^{3+}$ rich layer are antiparallel, has been put forward through X-ray magnetic circular dichroism $[10,12,21]$, and has been supported by theoretical studies using both density functional theory and Monte Carlo simulations as well as inelastic scattering experiments [21-23]. Apart from this 2D spin arrangement in the bilayer, Mulders et al. have suggested a long range AFM order along the $\mathrm{c}$ direction that facilitates the long range order of electric dipole moment, suggesting the presence of spin-charge coupling [12]. A magnetic field vs. temperature phase diagram above $180 \mathrm{~K}$ has been 
recently proposed, which reveals that magnetic field promotes the FIM order from the degenerate AFM-FIM phases [20].

In this work, we present a neutron diffraction experiment on single crystal $\mathrm{LuFe}_{2} \mathrm{O}_{4+\delta}$ under an applied magnetic field along the Ising direction in the temperature range 5-275 K. The striking effect of the magnetic field on the magnetic structure from our experiment together with previous works brings new insights into the complex magnetic field versus temperature phase diagram and reveals clear evidence for a field-induced coupling between the spin and charge orderings.

\section{Results}

Figure 2a shows the magnetic susceptibility of $\mathrm{LuFe}_{2} \mathrm{O}_{4+\delta}$ under zero field cooled (ZFC) and field cooled (FC) conditions measured by applying a magnetic field of $1 \mathrm{~T}$ parallel to the $c$-axis. In accordance with previous results, the ferrimagnetic order appears below $\mathrm{T}_{\mathrm{N}}=240 \mathrm{~K}$ [1]. A significant irreversibility between the ZFC and FC data can be seen at around $185 \mathrm{~K}$. However, the second magnetic phase transition at $175 \mathrm{~K}$ reported in Reference [14] is absent in this sample. The magnetization measurements are consistent with a sample containing a slight oxygen excess [1,3]. As shown in the inset of Figure 2a, the magnetic hysteresis loop at $200 \mathrm{~K}$ is indicative of a net spontaneous moment.

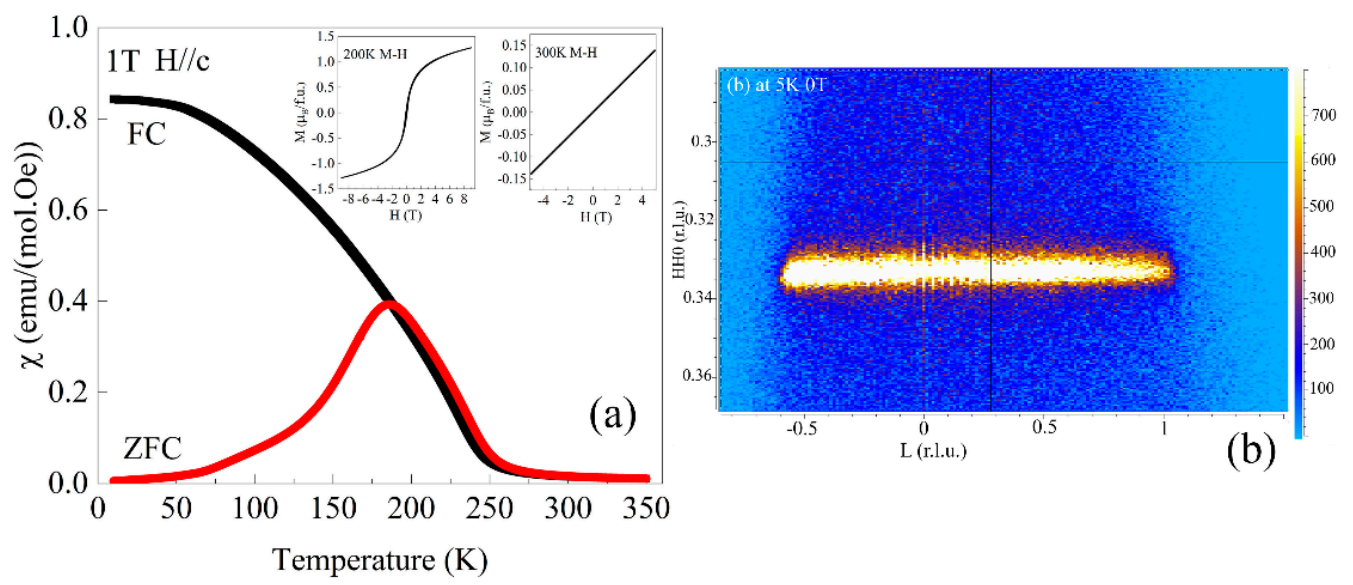

Figure 2. (a) Magnetic susceptibility of $\mathrm{LuFe}_{2} \mathrm{O}_{4+\delta}$ measured with an applied field of $1 \mathrm{~T}$ parallel to the $c$-axis. Insets show the magnetic hysteresis loops at 200 and $300 \mathrm{~K}$. (b) The strong spin correlations along $1 / 31 / 3 \mathrm{~L}$ rod at $5 \mathrm{~K}$ and $0 \mathrm{~T}$. The absence of intensity when $\mathrm{L}<-0.6$ and $>1$ is an artefact of the magnet coverage.

The neutron diffraction data reveals strong magnetic diffuse scattering, as can be seen in Figure $2 \mathrm{~b}$. This scattering is sharp in the HK0 plane, located in the $1 / 31 / 3$ position, and diffuse along the $\mathrm{L}$ direction forming a rod in the reciprocal space. This scattering persists down to $5 \mathrm{~K}$ in the zero field and is indicative of 2D magnetic correlations in the $\mathrm{FeO}_{5}$ triangular bilayers. As shown in Figure 3a, the integrated intensity of the reciprocal space cut along the 1/3 1/3 L direction becomes more intense below $240 \mathrm{~K}$, in good agreement with the magnetization measurement. Remarkably, a long range spin order associated with a propagation vector $\mathbf{k}_{\mathbf{1}}=0$ and coupled to the 2D spin correlations is unveiled below $240 \mathrm{~K}$, and is characterized by sharp magnetic contribution to the 110, 011 and -221 nuclear reflections, as shown in Figure $3 \mathrm{~b}$. This $\mathbf{k}_{\mathbf{1}}=0$ component is related to a ferromagnetic contribution with the $R-3 m^{\prime}$ symmetry as can be appreciated in Figure A1 (an AFM arrangement with the $R-3^{\prime} m^{\prime}$ symmetry would result in null magnetic intensity in the HK0 plane reflections). A net moment for a single triangular unit of Ising spins is naturally associated with up-up-down (down-down-up) spin configurations. The presence of the coherent scattering with $\mathbf{k}_{\mathbf{1}}=0 \mathrm{implies,}$ in the simplest case, an averaging between up-up-down, up-down-up and down-up-up configurations for each triangle. Practically, it means that the numbers of spins with up and down polarization are not equal 
in each triangular layer, resulting in a net ferrimagnetic moment. An illustrative example of such a ferrimagnetic layer is shown in Figure 3c. The layers are then stacked along the $c$-axis with a random shift within the $(a b)$ plane. The strong $2 \mathrm{D}$ magnetic scattering rod together with the 3D ferromagnetic component lead to a physical picture of spin ordering in $\mathrm{LuFe}_{2} \mathrm{O}_{4+\delta}$ from $5 \mathrm{~K}$ to $270 \mathrm{~K}$ in which the lack of spin correlations along the $\mathrm{c}$ direction coexists with the long range Ising ferrimagnetic order in each triangular monolayer.
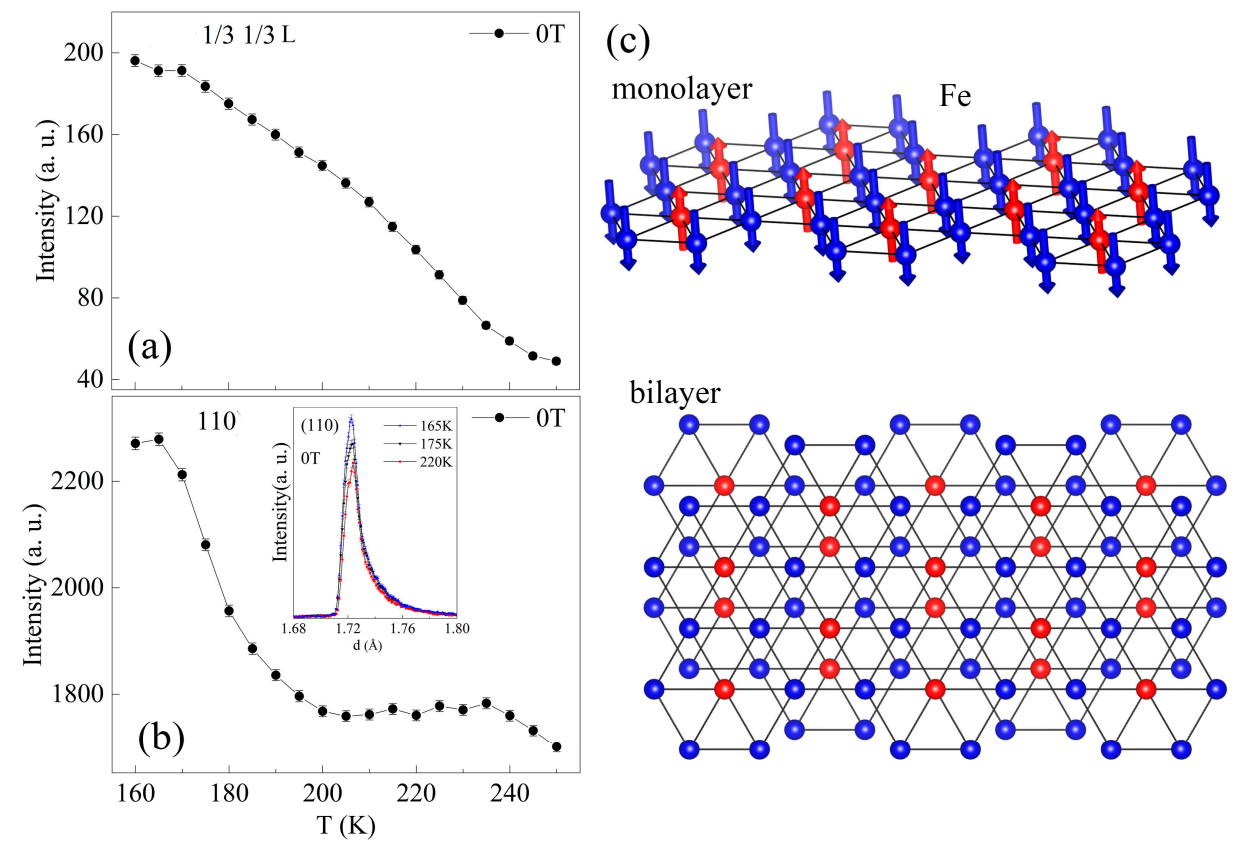

Figure 3. Temperature dependence of the integrated intensities of (a) the diffuse scattering at $1 / 3$ $1 / 3 \mathrm{~L}$ and (b) the 110 reflection at $150 \mathrm{~K}$. The inset shows the 110 reflection at 165, 175 and $220 \mathrm{~K}$. (c) Schematic drawing of a spin configuration with a net ferrimagnetic moment within a layer (upper panel) and a stacking of two adjacent monolayers into a bilayer (lower panel). Red (resp. blue) spheres represent up (resp. down) spins for the lower diagram.

The magnetic field dependence of neutron magnetic diffraction at $150 \mathrm{~K}$ is shown in Figure $4 \mathrm{a}-\mathrm{c}$. The application of an external field along the $c$ direction induces a strong change in the distribution of the diffuse scattering. Firstly, there is a strong intensity increase around the $1 / 31 / 31 / 2$ position (Figure 4c), the scattering is peaked at this position but it is still not resolution limited, indicating a finite correlation length. Moreover, a weaker peak-like feature is observed around the $1 / 31 / 3$ 0 satellite reflection and interestingly it is no longer centred at the commensurate position in the HK0 plane but is slightly off (as can be appreciated from the inset of Figure 4a,b), indicating the development of incommensurate interactions within the Fe layers. A similar situation is observed in the diffuse scattering at the $2 / 32 / 3 \mathrm{~L}$ position. The observation of Bragg reflections at the $1 / 31 / 31 / 2$ and $2 / 32 / 31 / 2$ as well as other non-integer $\mathrm{L}$ positions clearly indicates that the application of an external field promotes $3 \mathrm{D}$ spin correlations between the $\mathrm{FeO}_{5}$ triangular bilayers with a propagation vector $\mathbf{k}_{2}=(1 / 3,1 / 3,3 / 2)$ with respect to the parent $R-3 m$ unit cell. It should be pointed out that this propagation vector is identical to the structural modulation associated with the charge ordering reported in Reference [6] and confirmed in the present study, as can be appreciated by the observation of charge satellite reflections in the high $\mathrm{Q}$ region in Figure A2. As presented in Figure $4 \mathrm{~d}$,e, the temperature dependence of integrated intensities of $1 / 31 / 30$ and $1 / 31 / 31 / 2$ satellite reflections under $5 \mathrm{~T}$ shows the onset of spin ordering at $260 \mathrm{~K}$, slightly higher than that under $0 \mathrm{~T}$. 

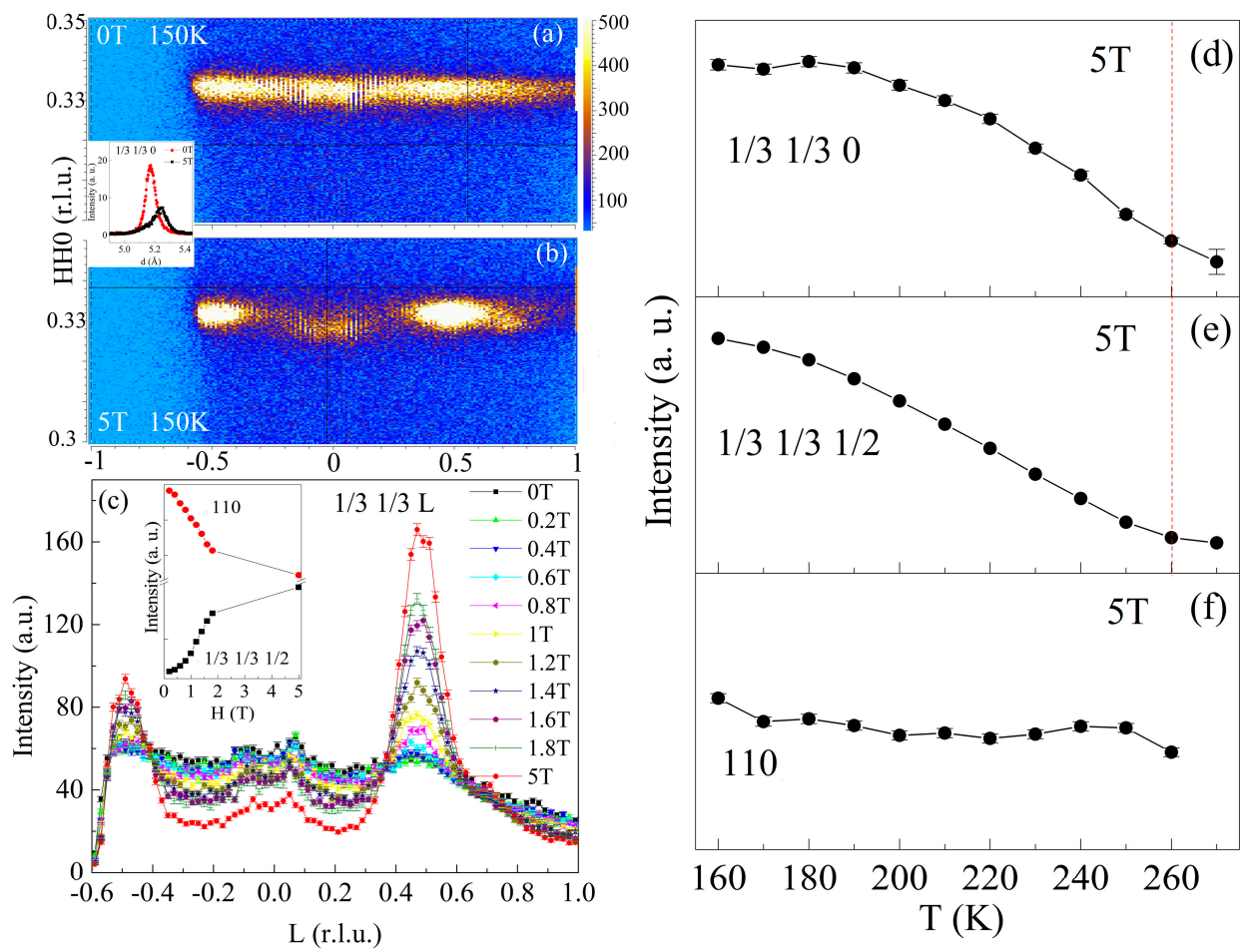

Figure 4. (a,b) Neutron scattering rod along $1 / 31 / 3 \mathrm{~L}$ direction under 0 and $5 \mathrm{~T}$ at $150 \mathrm{~K}$. The inset shows the incommensurate character of $1 / 31 / 30$ reflection when magnetic field of $5 \mathrm{~T}$ is applied along the c-axis. (c) Magnetic field dependence of neutron scattering along 1/3 1/3 L direction at $150 \mathrm{~K}$. The inset demonstrates the magnetic field dependence of the integrated intensities around the $1 / 31 / 31 / 2$ position and the 110 reflections at $150 \mathrm{~K}$. (d-f) Temperature dependencies of the integrated intensities of the $1 / 31 / 30,1 / 31 / 31 / 2$ and 110 reflections at $150 \mathrm{~K}$ under $5 \mathrm{~T}$.

Another outstanding consequence of the magnetic field is the dramatic decrease of integrated intensities of 110, 011 and -221 reflections. As seen in the inset of Figure 4c, the intensity of 110 peak monotonically decreases with increasing magnetic field. Under a magnetic field of $5 \mathrm{~T}$, as shown in Figure $4 \mathrm{f}$, there is no obvious variation of the integrated intensity of the 110 reflection with temperature. These observations show that the external magnetic field suppresses the $\mathbf{k}_{\mathbf{1}}$-related component. It is also important to stress that the magnetic scattering corresponding to the $\mathbf{k}_{\mathbf{2}}$ propagation vector cannot simply be ascribed to a redistribution of the diffuse scattering intensity but instead includes an extra component, likely coming from the magnetic scattering related to the $\mathbf{k}_{\mathbf{1}}$ propagation vector in zero field.

\section{Discussion}

The experimentally observed behaviour can be understood as a magnetic-field-imposed coupling between the spin and charge degrees of freedom. The component of the magnetic field along the $c$-axis transforms as the time-odd, one-dimensional representation $\Gamma_{2}{ }^{+}\left(H_{-}\right)$of the parent $R-3 m$ space group. The symmetry of the charge order cannot be uniquely identified from the available diffraction data and the only solid experimental evidence is that the propagation vector for this type of distortion is $(1 / 3$, $1 / 3,3 / 2)$, in agreement with other experimental results [6]. There are two six-dimensional irreducible representations associated with this propagation vector whose matrix operators for the generators of the $R-3 m$ space group are specified in Table 1 . Whichever of them is responsible for the symmetry breaking related to the charge ordering, there is always a way to form a tri-linear free-energy coupling term with a time-odd quantity (magnetic order) with the same periodicity as the charge ordering. 
Specifying the relevant order parameters as $\left(\eta_{+}, \eta^{*}, \xi_{+}, \xi^{*}, \rho_{+}, \rho^{*}\right)$ for the charge ordering and $\left(\eta_{-}\right.$, $\left.\eta^{*}, \xi_{-}, \xi^{*}, \rho_{-}, \rho^{*}\right)$ for the field-induced magnetic order, the free-energy invariant reads:

$$
H_{-}\left(\eta-\xi^{*}+\xi_{-} \rho^{*}+\rho_{-} \eta^{*}+\eta_{+} \xi^{*}+\xi_{+} \rho^{*}+\rho_{+} \eta^{*}\right)
$$

Table 1. Irreducible representations for generating symmetry elements of the $R-3 m$ space group, associated with the propagation vector $\mathbf{k}_{\mathbf{2}}=(1 / 3,1 / 3,3 / 2)$.

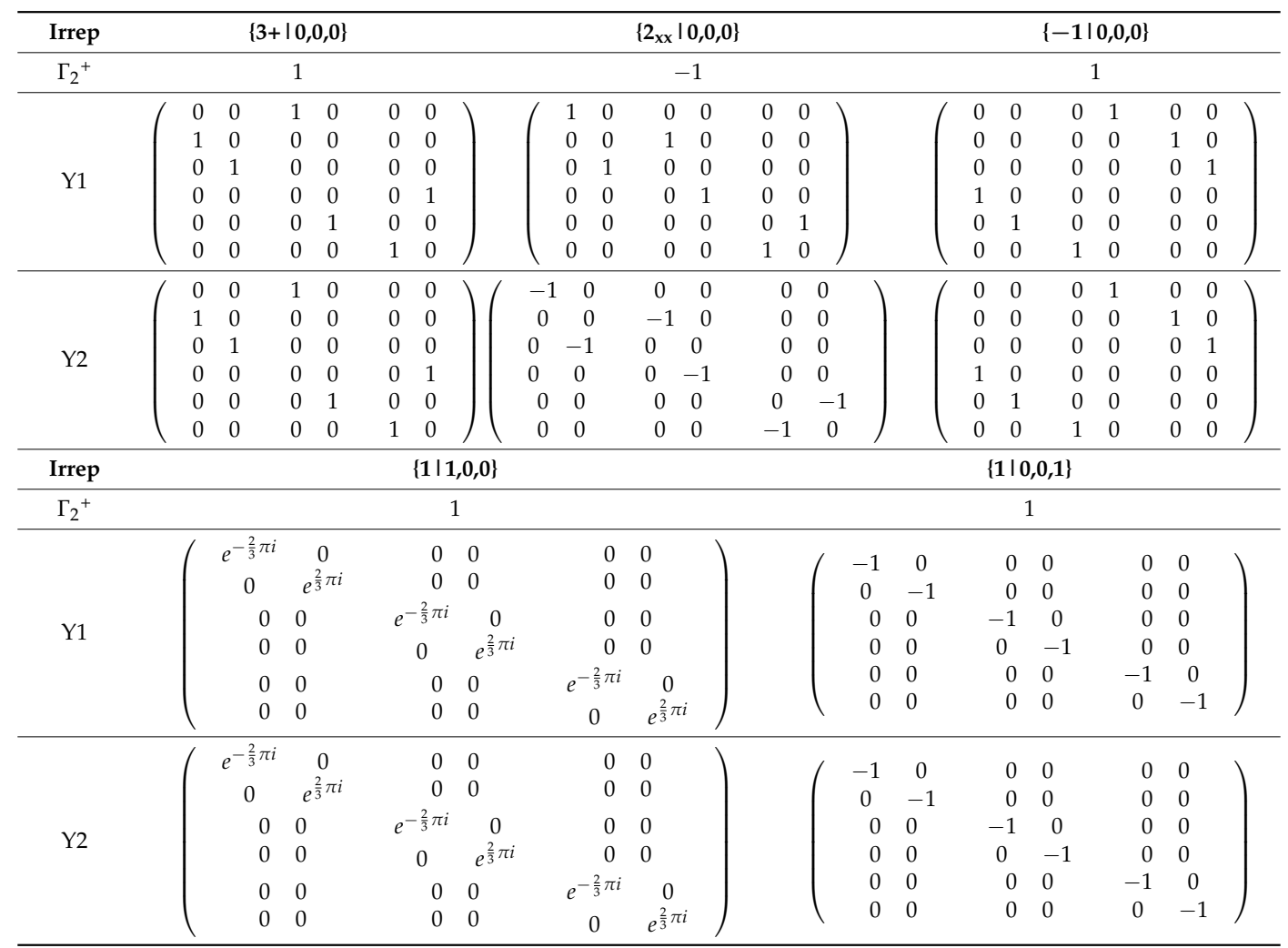

The subscripts and asterisk indicate time-parity and complex conjugation, respectively. If the charge ordering transforms as the time-even $\mathrm{Y} 1$ representation, then the field-induced magnetic ordering must belong to the time-odd $\mathrm{Y} 2$ representation. On the contrary, if the time-even $\mathrm{Y} 2$ is the relevant representation for the charge ordering, then the time-odd $Y 1$ describes the transformational properties of the induced magnetic order. Thus the condition to couple the charge and spin degrees of freedom in the applied magnetic field is that these order parameters must be transformed by the different representations. At a microscopic level, this implies that the combination of charge and spin orderings with the symmetries of the $\mathrm{Y} 1$ and $\mathrm{Y} 2$ representations should result in magnetic structures with uncompensated (ferrimagnetic) moments which can be coupled to the external magnetic field. Another conclusion arising from the symmetry consideration is that the coupling invariant does not vanish only if at least two (out of the three) arms of the (1/3,1/3,3/2)-propagation vector star are involved. Actually the term is maximal when the full star participates in the symmetry breaking and, therefore, if the charge ordering taking place in the zero-field exploits only a single arm (as for instance it was suggested in Reference [7]), then the application of the magnetic field should result in a re-distribution of the charge, making the ordering multi- $k$. Unfortunately, the current geometry of the diffraction experiment and the restrictions coming from the magnet do not allow us to verify this conclusion.

Finally, it is worth noting that following the discussion presented above, the presence of magnetic scattering in the incommensurate position close to $1 / 31 / 30$ might indicate the existence of a minor 
fraction of incommensurate charge ordered phase. We however do not have any experimental evidence for a structural modulation associated with this periodicity in our sample.

\section{Experiments and Methods}

The sample used here was cleaved from high-quality single crystal grown by the floating zone method, and the detailed experimental procedure can be found in Reference [22].

Magnetic susceptibility was measured using a vibrating sample magnetometer (VSM) (Physical Property Measurement System, Quantum Design, San Diego, CA, USA) between $10 \mathrm{~K}$ and $350 \mathrm{~K}$ under magnetic field of $1 \mathrm{~T}$ under both zero-field-cooled (ZFC) and field-cooled (FC) conditions. Magnetic hysteresis loops were measured at 200 and $300 \mathrm{~K}$.

Magnetic-field dependent single crystal neutron diffraction experiments were carried out at the ISIS pulsed neutron and muon facility of the Rutherford Appleton Laboratory (UK), on the WISH diffractometer [24] located at the second target station. The single crystal $(\sim 0.15 \mathrm{~g})$ was mounted in a vertical field superconducting cryomagnet with magnetic field up to $13.6 \mathrm{~T}$ and measured over the temperature range of $5-270 \mathrm{~K}$ with magnetic field applied along the $c$-axis. Group theoretical calculations were done using ISODISTORT [25] and Bilbao Crystallographic Server (Magnetic Symmetry and Applications) software [26]. Simulations of the neutron single crystal data were performed with the help of the Jana2006 software [27].

\section{Conclusions}

We investigated the effect of magnetic field on spin correlations in the charge ordered single crystal of $\mathrm{LuFe}_{2} \mathrm{O}_{4+\delta}$ through magnetization measurement and neutron diffraction. In the absence of a magnetic field, the spin and charge subsystems are effectively decoupled. The spin forms $2 \mathrm{D}$ correlations featured by the magnetic diffuse scattering rod in the $1 / 31 / 3 \mathrm{~L}$ direction and a 3D ferromagnetic component below $\mathrm{T}_{\mathrm{N}}=240 \mathrm{~K}$. An external magnetic field suppresses the diffuse scattering and promotes $3 \mathrm{D}$ spin correlations with the propagation vector $\mathbf{k}_{2}=(1 / 3,1 / 3,3 / 2)$. This periodicity is common for both the magnetic and charge-ordered sublattices and a combination of these experimental results with symmetry consideration provides evidence of a magnetic field imposed coupling between the spin and charge degrees of freedom.

Acknowledgments: Lei Ding thanks support from the Rutherford International Fellowship Programme (RIFP). This project has received funding from the European Union's Horizon 2020 research and innovation programme under the Marie Skłodowska-Curie Grant Agreements No. 665593 awarded to the Science and Technology Facilities Council. The work at the University of Warwick was supported by EPSRC, UK through Grant M028771/1. We thank the support received during material characterizations in the Materials characterization laboratory at the ISIS facility.

Author Contributions: Pascal Manuel, Dmitry D. Khalyavin and Lei Ding conceived the work; Andrew T. Boothroyd, Dharmalingam Prabhakaran and Geetha Balakrishnan performed single crystal growth; Pascal Manuel and Dmitry D. Khalyavin performed the neutron diffraction experiments; Lei Ding, Fabio Orlandi, Dmitry D. Khalyavin and Pascal Manuel analyzed the neutron data; Lei Ding also contributed to the magnetization measurements; Lei Ding, Fabio Orlandi, Dmitry D. Khalyavin and Pascal Manuel wrote the paper.

Conflicts of Interest: The authors declare no conflict of interest. 


\section{Appendix A}
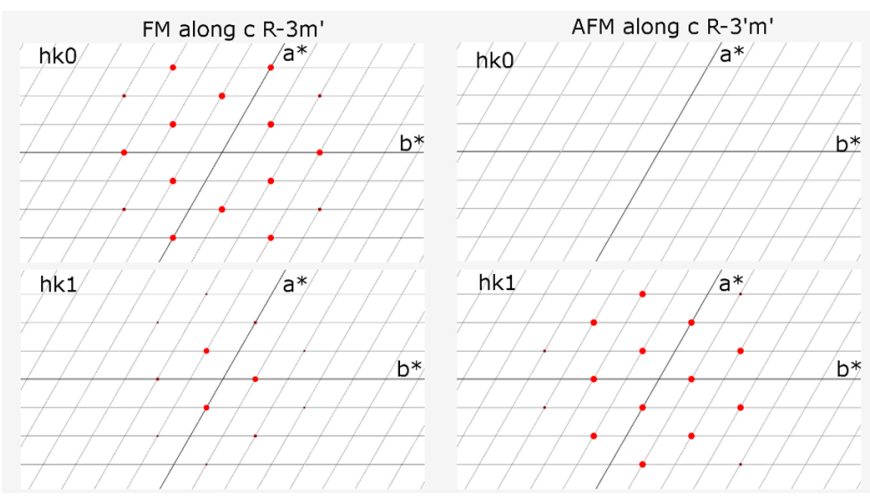

Figure A1. Simulated magnetic reflections in HK0 (Top) and HKL (Bottom) plane for a ferromagnetic (Left) and antiferromagnetic order (Right) along the c direction, the radius and the brightness of the reflections are an indication of the magnetic structure factor. The simulations are performed with the Jana2006 software.

(a)

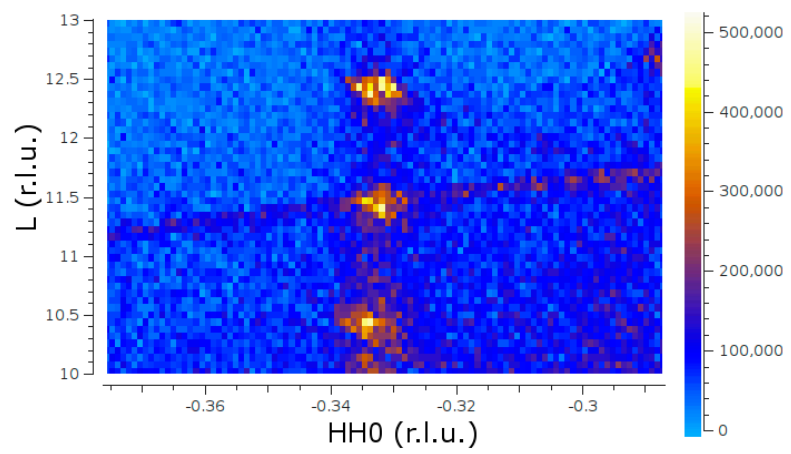

(b)

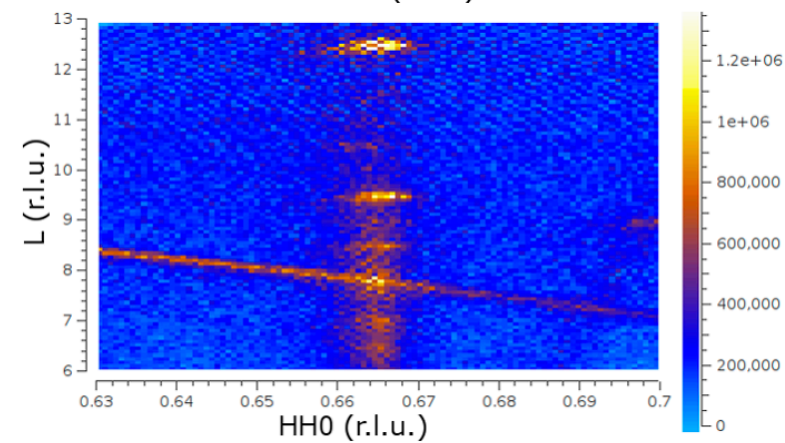

Figure A2. Neutron scattering in the high $\mathrm{Q}$ region at the (a) $1 / 31 / 3 \mathrm{~L}$ and (b) $2 / 32 / 3 \mathrm{~L}$ positions showing the presence of charge ordering satellite reflections ascribable to the $\mathbf{q}=(1 / 3,1 / 3,3 / 2)$ modulation vector.

\section{References}

1. Iida, J.; Tanaka, M.; Nakagawa, Y.; Funahashi, S.; Kimizuka, N.; Takekawa, S. Magnetization and spin correlation of two-dimensional triangular antiferromagnet $\mathrm{LuFe}_{2} \mathrm{O}_{4}$. J. Phys. Soc. Jpn. 1993, 62, 1723-1735. [CrossRef]

2. Yamada, Y.; Kitsuda, K.; Nohdo, S.; Ikeda, N. Charge and spin ordering process in the mixed-valence system $\mathrm{LuFe}_{2} \mathrm{O}_{4}$ : Charge ordering. Phys. Rev. B 2000, 62, 12167-12174. [CrossRef] 
3. Ikeda, N.; Ohsumi, H.; Ohwada, K.; Ishii, K.; Inami, T.; Kakurai, K.; Murakami, Y.; Yoshii, K.; Mori, S.; Horibe, $\mathrm{Y}$; e et al. Ferroelectricity from iron valence ordering in the charge-frustrated system $\mathrm{LuFe}_{2} \mathrm{O}_{4}$. Nature 2005, 436, 1136-1138. [CrossRef] [PubMed]

4. Nagano, A.; Naka, M.; Nasu, J.; Ishihara, S. Electric polarization, magnetoelectric effect, and orbital state of a layered iron oxide with frustrated geometry. Phys. Rev. Lett. 2007, 99, 217202. [CrossRef] [PubMed]

5. Xiang, H.J.; Whangbo, M.H. Charge order and the origin of giant magnetocapacitance in $\mathrm{LuFe}_{2} \mathrm{O}_{4}$. Phys. Rev. Lett. 2007, 98, 246403. [CrossRef] [PubMed]

6. Angst, M.; Hermann, R.P.; Christianson, A.D.; Lumsden, M.D.; Lee, C.; Whangbo, M.H.; Kim, J.W.; Ryan, P.J.; Nagler, S.E.; Tian, W.; et al. Charge order in $\mathrm{LuFe}_{2} \mathrm{O}_{4}$ : Antiferroelectric ground state and coupling to magnetism. Phys. Rev. Lett. 2008, 101, 227601. [CrossRef] [PubMed]

7. De Groot, J.; Mueller, T.; Rosenberg, R.A.; Keavney, D.J.; Islam, Z.; Kim, J.W.; Angst, M. Charge order in $\mathrm{LuFe}_{2} \mathrm{O}_{4}$ : An unlikely route to ferroelectricity. Phys. Rev. Lett. 2012, 108, 187601. [CrossRef] [PubMed]

8. Ikeda, N.; Nagata, T.; Kano, J.; Mori, S. Present status of the experimental aspect of $\mathrm{RFe}_{2} \mathrm{O}_{4}$ study. J. Phys. Condens. Matter 2015, 27, 053201. [CrossRef] [PubMed]

9. Xu, X.S.; De Groot, J.; Sun, Q.C.; Sales, B.C.; Mandrus, D.; Angst, M.; Litvinchuk, A.P.; Musfeldt, J.L. Lattice dynamical probe of charge order and antipolar bilayer stacking in $\mathrm{LuFe}_{2} \mathrm{O}_{4}$. Phys. Rev. B 2010, 82, 014304. [CrossRef]

10. Ko, K.T.; Noh, H.J.; Kim, J.Y.; Park, B.G.; Park, J.H.; Tanaka, A.; Kim, S.B.; Zhang, C.L.; Cheong, S.W. Electronic origin of giant magnetic anisotropy in multiferroic $\mathrm{LuFe}_{2} \mathrm{O}_{4}$. Phys. Rev. Lett. 2009, 103, 207202. [CrossRef] [PubMed]

11. Wen, J.S.; Xu, G.Y.; Gu, G.D.; Shapiro, S.M. Magnetic-field control of charge structures in the magnetically disordered phase of multiferroic $\mathrm{LuFe}_{2} \mathrm{O}_{4}$. Phys. Rev. B 2009, 80, 020403. [CrossRef]

12. Mulders, A.M.; Bartkowiak, M.; Hester, J.R.; Pomjakushina, E.; Conder, K. Ferroelectric charge order stabilized by antiferromagnetism in multiferroic $\mathrm{LuFe}_{2} \mathrm{O}_{4}$. Phys. Rev. B 2011, 84, 140403(R). [CrossRef]

13. Harris, A.B.; Yildirim, T. Charge and spin ordering in the mixed-valence compound $\mathrm{LuFe}_{2} \mathrm{O}_{4}$. Phys. Rev. $B$ 2010, 81, 134417. [CrossRef]

14. Christianson, A.D.; Lumsden, M.D.; Angst, M.; Yamani, Z.; Tian, W.; Jin, R.; Payzant, E.A.; Nagler, S.E.; Sales, B.C.; Mandrus, D. Three-dimensional magnetic correlations in multiferroic $\mathrm{LuFe}_{2} \mathrm{O}_{4}$. Phys. Rev. Lett. 2008, 100, 107601. [CrossRef] [PubMed]

15. Wang, F.; Kim, J.; Kim, Y.; Gu, G.D. Spin-glass behaviour in in $\mathrm{LuFe}_{2} \mathrm{O}_{4+\delta}$. Phys. Rev. B 2009, 80, 024419. [CrossRef]

16. Bourgeois, J.; Andre, G.; Petit, S.; Robert, J.; Poienar, M.; Rouquette, J.; Elkaim, E.; Hervieu, M.; Maignan, A.; Martin, C.; et al. Evidence of magnetic phase separation in $\mathrm{LuFe}_{2} \mathrm{O}_{4}$. Phys. Rev. B 2012, 86, 024413. [CrossRef]

17. Hervieu, M.; Guesdon, A.; Bourgeois, J.; Elkaim, E.; Poienar, M.; Damay, F.; Rouquette, J.; Maignan, A.; Martin, C. Oxygen storage capacity and structural flexibility of $\mathrm{LuFe}_{2} \mathrm{O}_{4+x}(0 \leq x \leq 0.5)$. Nat. Mater. 2013, 13, 74-80. [CrossRef] [PubMed]

18. Bourgeois, J.; Hervieu, M.; Poienar, M.; Abakumov, A.M.; Elkaim, E.; Sougrati, M.T.; Porcher, F.; Damay, F.; Rouquette, J.; Van Tendeloo, G.; et al. Evidence of oxygen-dependent modulation in $\mathrm{LuFe}_{2} \mathrm{O}_{4}$. Phys. Rev. B 2012, 85, 064102. [CrossRef]

19. Xu, X.S.; Angst, M.; Brinzari, T.V.; Hermann, R.P.; Musfeldt, J.L.; Christianson, A.D.; Mandrus, D.; Sales, B.C.; McGill, S.; Kim, J.W.; et al. Charge order, dynamics, and magnetostructural transition in multiferroic $\mathrm{LuFe}_{2} \mathrm{O}_{4}$. Phys. Rev. Lett. 2008, 101, 227602. [CrossRef] [PubMed]

20. De Groot, J.; Marty, K.; Lumsden, M.D.; Christianson, A.D.; Nagler, S.E.; Adiga, S.; Borghols, W.J.H.; Schmalzl, K.; Yamani, Z.; Bland, S.R.; et al. Competing Ferri- and Antiferromagnetic phases in geometrically frustrated $\mathrm{LuFe}_{2} \mathrm{O}_{4}$. Phys. Rev. Lett. 2012, 108, 037206. [CrossRef] [PubMed]

21. Kuepper, K.; Raekers, M.; Taubitz, C.; Prinz, M.; Derks, C.; Neumann, M.; Postnikov, A.V.; De Groot, F.M.F.; Piamonteze, C.; Prabhakaran, D.; et al. Charge order, enhanced orbital moment, and absence of magnetic frustration in layered multiferroic $\mathrm{LuFe}_{2} \mathrm{O}_{4}$. Phys. Rev. Lett. 2009, 80, 220409(R).

22. Gaw, S.M.; Lewtas, H.J.; McMorrow, D.F.; Kulda, J.; Ewings, R.A.; Perring, T.G.; McKinnon, R.A.; Balakrishnan, G.; Prabhakaran, D.; Boothroyd, A.T. Magnetic excitation spectrum of $\mathrm{LuFe}_{2} \mathrm{O}_{4}$ measured with inelastic neutron scattering. Phys. Rev. B 2015, 91, 035103. [CrossRef]

23. Xiang, H.J.; Kan, E.J.; Wei, S.H.; Whangbo, M.H.; Yang, J.L. Origin of the Ising ferrimagnetism and spin-charge coupling in $\mathrm{LuFe}_{2} \mathrm{O}_{4}$. Phys. Rev. B 2009, 80, 132408. [CrossRef] 
24. Chapon, L.; Manuel, P.; Radaelli, P.G.; Benson, C.; Perrott, L.; Ansell, S.; Rhodes, N.J.; Raspino, D.; Duxbury, D.; Spill, E.; et al. Wish: The new powder and single crystal magnetic diffractometer on the second target station. Neutron News 2011, 22, 22-25. [CrossRef]

25. Campbell, B.J.; Stokes, H.T.; Tanner, D.E.; Hatch, D.M. ISODISPLACE: A web-based tool for exploring structural distortions. J. Appl. Cryst. 2006, 39, 607-614. [CrossRef]

26. Perez-Mato, J.M.; Gallego, S.V.; Tasci, E.S.; Elcoro, L.; de la Flor, G.; Aroyo, M.I. Symmetry-based computational tools for magnetic crystallography. Annu. Rev. Mater. Res. 2015, 45, 217-248. [CrossRef]

27. Petricek, V.; Dusek, M.; Palatinus, L. Crystallographic computing system JANA2006: General features. Z. Kristallogr. Cryst. Mater. 2014, 229, 345-352. [CrossRef]

2018 by the authors. Licensee MDPI, Basel, Switzerland. This article is an open access article distributed under the terms and conditions of the Creative Commons Attribution (CC BY) license (http://creativecommons.org/licenses/by/4.0/). 